\title{
Knowledge, Attitude and Practices of Dental Professionals in Rwanda towards the Benefits and Applications of Teledentistry
}

\author{
Julienne Murererehe ${ }^{1 *}$, Peace Uwambaye ${ }^{1}$, Moses Isyagi ${ }^{1}$, Theogene Nyandwi ${ }^{2}$, Kato Njunwa ${ }^{3}$ \\ ${ }^{1}$ School of Dentistry, College of Medicine and Health Sciences, University of Rwanda \\ ${ }^{2}$ Coordinator of Research and innovations, Directorate of research, College of medicine and Health sciences, University of Rwanda \\ ${ }^{3}$ University Director of Research, Innovation and Post Graduate Studies, University of Rwanda
}

\begin{abstract}
Background: Currently, the use of teledentistry is still low in developing countries. In Rwanda, the state of teledentistry is undocumented. Without knowledge and positive attitude of dental practitioners towards teledentistry, it will not be easy to implement teledentistry innovations. Methods: To assess the knowledge, attitude and practices of dental professionals towards teledentistry, a cross-sectional study was conducted in Kigali-Rwanda on 103 dental professionals. Results: The majority of respondents had good knowledge and positive attitude (88\%) towards the benefits and application of teledentistry. However, the practice of teledentistry being done by dental professionals is still at low level where only seven $(6.8 \%)$ of dental professionals are using videoconference for health related purpose intercommunication. Also only $13(12.6 \%)$ and eight $(7.8 \%)$ were sharing digital x-ray and digital photographs as the reason for intercommunication respectively. The knowledge, attitude and daily internet access were significantly positively associated with practice $(p<0.05)$. Also knowledge of dental professionals towards the benefits and application of teledentistry was significantly and positively associated with their attitude teledentistry $(p<0.05)$. Conclusion: The results revealed that dental professionals are knowledgeable and have positive attitude towards teledentistry. Again, dental professionals overall believe that teledentistry is good and it has to be integrated into the current dental practice. However, the practice of teledentistry is still at low level in Rwanda compared to other advanced applications of teledentistry done in developed countries.
\end{abstract}

Keywords: Teledentistry, attitude, knowledge, practice, dental professionals

\section{Background}

Oral health disparities are a global issue with most dental specialists located in urban areas. Limited number of dental professionals in rural area leaves people without access to quality dental care in those areas (Berndt, Leone, \& King, 2008). Information technology has allowed quick and efficient information transmission. The development of communication programs has allowed the appearance of a global society and electronic interconnection with no geographical boundary (Costa, Silva, \& Pereira, 2011). In the health sector, several technological methods referred to as telehealth, are being used. They allow interaction between patients and healthcare providers at a distance in different areas (Costa et al., 2011).

The goal of telehealth is broad and it allows continuing education, research, exchanging of necessary information for diagnosis, treatment, and to predict and prevent diseases from occurring. Additionally, telehealth is considered to be a new tool to overcome cultural, social economic and geographic barriers. Its primary benefits include improved primary health care, access to health care, increased availability of resources for education and health professional information (Costa et al., 2011).

Most low income countries have started to use telehealth as a mean to overcome challenges related to shortage of specialists, lack of infrastructures, limited clinical services and inexperienced primary health care providers. Telehealth technologies have increasingly been put into practice in dentistry since it was first launched in 1994 in the provision of oral health services and it is known as teledentistry (Glassman, Helgeson, \& Kattlove, 2012a).
One of teledentistry role is to reduce the gap between the increased population demand for better oral health care and existing financial resources (Nagarajappa et al., 2013a).

New innovations in research are also increasing in dentistry and such innovations are coming through teledentistry (Sanjeev, \& Shushant K, 2011a). However, the research in teledentistry has lagged behind when compared with general medicine (Mihailovic, Miladinovic, \& Vujicic, 2011). The studies conducted in teledentistry, were mainly done in developed countries thereby leaving a wide gap in knowledge in low income countries (Mariño \& Ghanim, 2013).

Rwanda, in its vision 2020, intended to strongly consider Information and Communication Technology (ICT) as enabler and key driver for broad socio-economic development in the areas of healthcare, education and knowledge based society (Ignace Gatare, 2011). Shortage of dentists and dental therapists, numbering only 92 for 10 million people, however, remains a challenge address on all fronts (Seymour et al., 2013a). Fortunately, Rwanda has been a pioneer in national initiatives to integrate technology into its expanding health care system. Open Medical Record System (OpenMRS), TRACnet and TRACPlus are a comprehensive data entry, storage, access, and sharing system created in Rwanda by the Treatment and Research AIDS Center (TRAC), and they monitor on monthly basis infectious diseases including Human Immunodeficiency Virus/Acquired Immunodeficiency Syndrome (HIV/AIDS), tuberculosis (TB), and Malaria, drug, medical supply management system, Management Information Systems (HMIS). HMIS integrates data collection, processing, 
reporting, and use of the information for programmatic decision-making. E-Learning uses ICT in instruction of certificate level (A2) nurses to upgrade to Advanced Diploma (A1) level and Telemedicine (Frasier, May, \& Wanchoo, 2008). Although teledentistry initiatives have already started in Rwanda, there is no documented evidence showing the knowledge, attitude and practices of dental professionals towards teledentistry.

This study aimed to determine the knowledge and attitude of dental professionals in Kigali towards the benefits and applications of teledentistry, to identify the perceived challenges of dental professionals in Kigali to the use of teledentistry, to identify teledentistry practices of dental professionals in Kigali.

\section{Methods}

\section{Study area}

The study was conducted in different public and private dental clinics or institutions in Kigali city, i.e. University of Rwanda- College of Medicine and Health Sciences (URCMHS) both dental clinic and school of dentistry; Kigali Teaching Hospital (CHUK), Rwanda Military Hospital (RMH), Muhima Hospital (MH), Faith dental clinic, African Dental Aid (ADA) clinic.

\section{Study design, study population, sample size and sampling strategy}

A cross-sectional study using quantitative method was conducted on 157 pre-determined dental professionals in different public and private dental clinics or institutions found in Kigali city. All dental professionals working or studying in dentistry in Kigali city were considered due to limited number of dental professionals. 103 dental professional participated in the study. A census method was used as a sampling strategy for dental professionals in Kigali city. Dental professionals who were not around during the time of data correction or dental professionals who did not consent to participate in the study were excluded from the study.

A dental professional is any of many professionals supporting or involved in dentistry such as dentists, dental students, dental educators, dental assistants and dental technicians (Ozkan Ata, \& Ozkan, 2009a).

A census method is a technique where data are collected from the whole population rather than a sample.

\section{Data collection methods and procedures.}

A self administered structured questionnaire was used to collect data regarding knowledge, attitude and practices of dental professionals towards teledentistry. The questionnaire was designed in English. The questionnaire was adapted, validated and modified from questionnaires of similar studies found in the literature (Cooper \& Engeswick, 2007; Nagarajappa et al., 2013a; Ozkan Ata, \& Ozkan, 2009a).

A pilot study with 10 students and five dental practitioners was done prior to the study to pre-test the questionnaire. The aim of the pilot study was to ensure the validity and reliability of the questionnaire and to measure the understanding of participants to ensure the acceptability of the questionnaire and the language clarity. The researcher provided research information sheet to inform both students and dental practitioners about the aim of the research before they answered the questionnaire.

\section{Data analysis}

Data were analysed using statistical software STATA version 13. The response format was based on 5-Point Likert scale for knowledge and attitude. Level of significance was fixed at $p \leq 0.05$. Frequencies, means and standard deviations as part of descriptive statistics were used to describe the data. One way ANOVA and Chi-square tests were performed to assess whether the knowledge and attitude varies according to socio-demographic characteristics or to each other and to assess their relationship with practice. Tables were used to present the results.

\section{Ethical consideration}

The scientific and ethical approval for conducting the research was granted by UR- CMHS Ethical Committee. Likewise, permission to collect data was obtained from different sites where data were collected. Informed consent form was signed by each participant willing to participate before answering the questionnaire. The participation in the study was on voluntary basis and confidentiality of the identity of the participants was assured by using codes instead of real names of participants on the questionnaire.

\section{Problems and limitation of the study}

During the study, the researcher encountered difficulty of getting permission to collect data in sites that didn't have ethical committee. Another problem was dental professionals working in more than one dental clinic. We considered only one site for those people to avoid the duplication of data. The study was conducted in Kigali city and may not be generalized to the whole country. Another limitation is that the population sampled was not categorized.

\section{Results}

Out of 157 pre- determined dental professionals, 103 dental professionals participated in the study. Therefore, the rate of participation is $65.6 \%$. The remaining dental professional did not participate in the study. Some were not present at the time of data collection and others did not answer the questionnaire.

\section{Knowledge and attitude of dental professionals about the benefits and application of teledentistry}

Regarding the knowledge of dental professionals about benefits and application of teledentistry, respondents have shown good knowledge in general. Majority (the highest being $99(96.1 \%)$ and the lowest being $57(55.3 \%)$ of dental professionals agreed with all given items about the benefits and application of teledentistry. Regarding the attitude of dental professionals, majority (the highest being 82 (79.6\%) and the lowest being $57(55.3 \%)$ of dental professionals showed positive attitude regarding the benefits and application of teledentistry. However about $46(44.7 \%)$ of respondents disagreed with the item "I think teledentistry 
can reduce the costs of dental services and $43(41.7 \%)$ disagreed with the item "I think that dental examination done through teledentistry is as accurate as face to face consultation.

Table1. Knowledge and Attitude of Dental professionals towards teledentistry

\section{KNOWLEDGE ABOUT TELEDENTISTRY BENEFITS AND APPLICATIONS}

\begin{tabular}{|c|c|c|c|}
\hline & $\begin{array}{l}\text { Agree } \\
\mathrm{N}(\%)\end{array}$ & $\begin{array}{l}\text { Neutral } \\
\text { N (\%) }\end{array}$ & $\begin{array}{l}\text { Disagree } \\
\text { N (\%) }\end{array}$ \\
\hline Knowledge about teledentistry concept & $99(96.1)$ & $4(3.9)$ & $0(0)$ \\
\hline Use in every branch of dentistry & $92(89.3)$ & $5(4.85)$ & $6(5.83)$ \\
\hline Use for education and training of primary healthcare dentists. & $88(85.44)$ & $8(7.77)$ & $7(6.8)$ \\
\hline decreasing the isolation of dental practitioners by providing peer contact specialist & $86(83.5)$ & $11(10.68)$ & $6(5.83)$ \\
\hline Useful in diagnosis and management of oral diseases & $87(84.5)$ & $11(10.68)$ & $5(4.85)$ \\
\hline Useful in early and easy consultation of oral- facial disorders with specialists. & $88(85.4)$ & $7(6.8)$ & $8(7.77)$ \\
\hline Make sound decisions regarding management of oral diseases and referral to appropriate dental specialists. & $90(87.4)$ & 10(9.71) & $3(2.91)$ \\
\hline Prevents doctors' mistakes and improves cost-effectiveness of dental treatment. & $77(75)$ & $15(14.56)$ & 11(10.68) \\
\hline $\begin{array}{l}\text { Used as a good tool for education to undergraduate students, post-graduate students and continuing } \\
\text { updates for dental practitioners. }\end{array}$ & $95(92.2)$ & 7.6.8) & $1(0.97)$ \\
\hline Increase the number of dental specialist trainees and specialists in areas where population are dispersed. & $83(80.6)$ & 12(11.69) & $8(7.77)$ \\
\hline
\end{tabular}

\section{ATTITUDE ABOUT TELEDENTISTRY BENEFITS AND APPLICATIONS}

\begin{tabular}{|c|c|c|c|}
\hline Teledentistry can save me time. & $82(79.6)$ & 11(10.68) & $10(9.71)$ \\
\hline Teledentistry can save me money. & $71(69)$ & $17(16.50)$ & $15(14.56)$ \\
\hline Using teledentistry, I will be able to monitor my patient's condition well. & $80(77.7)$ & $12(11.65$ & $11(10.68)$ \\
\hline Teledentistry can provide me a good understanding of the patient's oral health problem over the internet. & $83(80.6)$ & $11(10.68)$ & $9(8.74)$ \\
\hline Teledentistry will be a standard way of oral health care delivery & $77(75)$ & 16(15.53) & $10(9.71)$ \\
\hline Teledentistry is a convenient form of oral health care delivery which makes dental examination easier & $76(73.8)$ & $19(18.45)$ & $8(7.77)$ \\
\hline Teledentistry can be an addition to the regular care we provide. & $79(76.70)$ & $18(17.48)$ & $6(5.83)$ \\
\hline I think dental examination done through teledentistry is as accurate as face to face consultation & $60(58.3)$ & $19(18.45)$ & $24(23.3)$ \\
\hline I think teledentistry can reduce the costs of dental services & $57(55.3)$ & 19(18.45) & $27(26.21)$ \\
\hline $\begin{array}{l}\text { I think through videoconference ( a medium of teledentistry) students can be educated and staff be } \\
\text { trained effectively }\end{array}$ & $94(91.2)$ & $8(7.77)$ & $1(0.97)$ \\
\hline Overall believe of dental professionals about teledentistry benefits and application & $97(94.2)$ & $0(0)$ & $6(5.8)$ \\
\hline
\end{tabular}

\section{Perceived challenges to the use of Teledentistry.}

Majority of participants $78(75.8 \%$ ) agreed that in Rwanda, major challenges in teledentistry use are illiteracy, poverty (i.e. population below the poverty line) and lack of infrastructures. A large number of participants $83(80.6 \%)$ think that lack of awareness of teledentistry benefits and applications by dental professionals will be barriers to its use. However a large number of participants showed a negative attitude for the use of teledentistry. Many respondents $52(50.5 \%)$ thought that teledentistry can violate the patient privacy. About 50 (48.5\%) were worried about data entry mistakes. Forty-one $(40 \%)$ of dental professionals can't trust teledentistry equipment to work properly and $47(45.6 \%)$ think that teledentistry equipment will be difficult for them to use.

Table 2. Frequency of perceived challenges to the use of teledentistry

\begin{tabular}{|c|c|c|c|}
\hline \multirow{2}{*}{ PERCEIVED CHALLENGES TO THE USE OF TELEDENTISTRY } & \multicolumn{3}{|c|}{ PROPORTION } \\
\hline & Agree $\mathbf{N}(\%)$ & Neutral N (\%) & Disagree $\mathrm{N}(\%)$ \\
\hline I think teledentistry can violate the patient's privacy. & $52(50.5)$ & 21(20.39) & $30(29.13)$ \\
\hline I am worried about data entry mistakes. & $50(48.5)$ & 31(30.10). & $22(21.36)$ \\
\hline I can't trust teledentistry equipment to work. & $41(40)$ & $25(24.27)$ & $37(35.92)$ \\
\hline I think equipments of teledentistry will be difficult for me to use them. & $47(45.6)$ & $16(15.53)$ & $40(38.83)$ \\
\hline $\begin{array}{l}\text { In Rwanda, major challenges in teledentistry are illiterates, population below } \\
\text { the poverty line and lack of infrastructure. }\end{array}$ & $78(76)$ & $13(12.62)$ & $12(11.65)$ \\
\hline $\begin{array}{l}\text { I think lack of awareness about teledentistry benefits and applications by dental } \\
\text { professionals will be a barrier to its use. }\end{array}$ & $83(80.6)$ & $11(10.68)$ & $9(8.74)$ \\
\hline
\end{tabular}


Frequency of practices of dental professionals towards teledentistry.

Majority 87 (84.5\%) of dental professionals are communicating with peer dental professionals through internet or mobile phone. The results also revealed that highly used intercommunication method was email, 69 $(67 \%)$ while videoconference, 7 (6.8\%). Regarding reasons of intercommunication, majority of respondents indicated patient information sharing 43 (42\%), discussing patients' cases $40(39 \%)$, digital photographs sharing $8(7.8 \%)$ and digital $x$-ray image sharing $13(12.6 \%)$.

Table3. Practices of dental professionals towards teledentistry.

\begin{tabular}{lll}
\hline \multicolumn{1}{c}{ Practices of dental professionals } & Yes N (\%) & No N (\%) \\
\hline $\begin{array}{l}\text { Interdisciplinary consultation or communication with } \\
\text { peer dental professionals through internet or mobile } \\
\text { phone }\end{array}$ & $87(84.5 \%)$ & $15(14.5 \%)$ \\
Category of people with whom intercommunication is done & \\
Consultation with patients & $30(29.1 \%)$ & $73(70.9 \%)$ \\
Consultation with students & $52(50.5 \%)$ & $51(49.5 \%)$ \\
Consultation with educators & $29(28 \%)$ & $74(72 \%)$ \\
Consultation with specialists in the same field & $32(31 \%)$ & $71(69 \%)$ \\
Consultation with own peers & $26(25 \%)$ & $77(75 \%)$ \\
Consultation with insurance company & $4(4 \%)$ & $99(96 \%)$
\end{tabular}

Means of intercommunication among dental professionals for health related purpose

Usage of videoconference as a mean of intercommunication

$7(6.8 \%) \quad 96(93.2 \%)$

Usage of email as a mean of intercommunication $69(67 \%) \quad 34(33 \%)$

Phone call and sms

$21(20.4 \%) \quad 82(79.6 \%)$

Facebook, Linkedin, whatsapp $13(12.6 \%) \quad 90(87.4 \%)$

Reasons for intercommunication

Discussing a patients case as a reason of consultation $40(39 \%) \quad 63(61 \%)$

Patient information sharing as a reason of consultation $43(42 \%) \quad 60(58 \%)$ Getting opinion from an expert for a patient case as $\quad 26(25 \%) \quad 77(74.8 \%)$
reason of consultation

$23(22.3 \%) \quad 80(77.7 \%)$

Patient follow up as a reason of consultation

$37(36 \%) \quad 66(64 \%)$

Course material clarification as a reason of consultation

$8(7.8 \%) \quad 95(92.2 \%)$

consultation

$13(12.6 \%) \quad 90(87.4 \%)$

Sharing digital $\mathrm{x}$-ray images as a reason of consultation

Mean score of knowledge and attitude with socio-economic and demographic characteristics of dental professionals.

To assess the overall knowledge and attitude according to demographic data characteristics, calculation of the means and standard deviations to each category of sociodemographic characteristics was made. The calculation of mean knowledge or mean attitude was done by summing the scores of responses given. After all, One way ANOVA statistical analysis was performed and no association found between knowledge and attitude with socio-demographic data.

Table 4. Mean knowledge and attitude scores with socio-demographic characteristics and practice of dental professionals towards teledentistry.

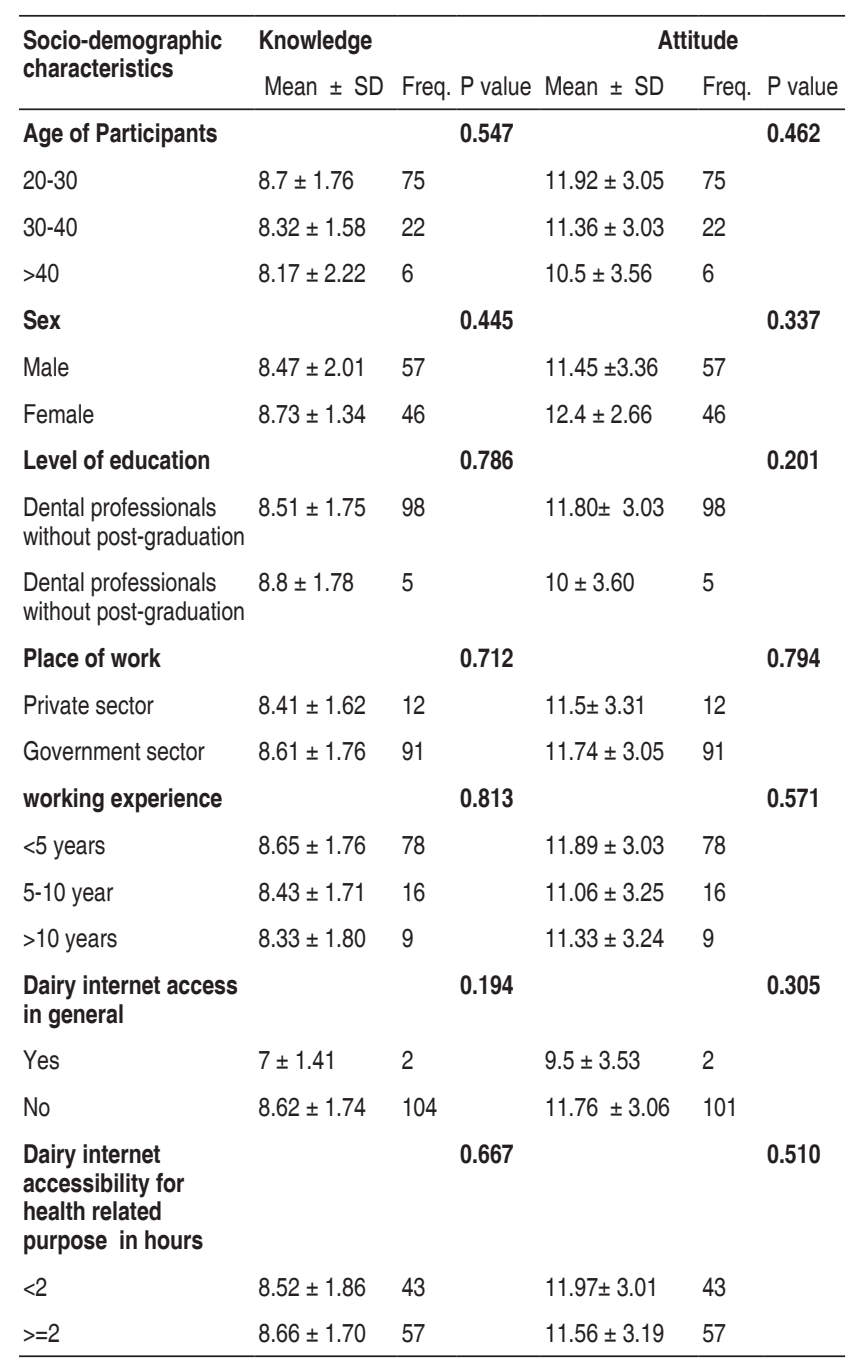

Statistical test applied: one way ANOVA.

* indicates statistically significant difference at $p \leq$ 0.05 .

\section{Association of knowledge and attitude scores.}

The proportion of knowledge and attitude scores have been calculated and

Chi square statistical analysis has been performed. The results revealed statistically significant association between knowledge and attitude $(P<0.05)$

\section{Association of socio-demographic data with practice of teledentistry.}

One way ANOVA statistical analysis was performed to see the relationship between socio- demographic characteristics 
with practice. The results revealed that the only predictor of practice was internet access in general $(P<0.05)$

Table5. Association of socio-demographic data with practices of teledentistry.

\begin{tabular}{|c|c|c|c|}
\hline \multirow[b]{2}{*}{ Socio-demographic characteristics. } & \multicolumn{3}{|c|}{ Practice } \\
\hline & Yes n (\%) & No $n(\%)$ & $\begin{array}{l}\text { Fisher's } \\
\text { exact } \\
\text { P value }\end{array}$ \\
\hline \multicolumn{4}{|l|}{ Age of Participants } \\
\hline $20-30$ & $63(70.79)$ & $12(85.71)$ & \\
\hline $30-40$ & $20(22.47)$ & $2(14.29)$ & 0.592 \\
\hline $\begin{array}{l}>40 \\
\text { Sex }\end{array}$ & $6(6.74)$ & $0(0.00)$ & \\
\hline Male & $49(55.06)$ & $8(57.14)$ & 1.000 \\
\hline Female & $40(44.94)$ & $6(42.86)$ & \\
\hline $\begin{array}{l}\text { Level of education } \\
\text { Dental professionals without post- } \\
\text { graduation } \\
\text { Dental professionals without post- } \\
\text { graduation }\end{array}$ & $\begin{array}{l}84(94.38) \\
5(5.62)\end{array}$ & $\begin{array}{l}14(100.00) \\
0(0.00)\end{array}$ & 1.000 \\
\hline Place of work & & & 0.363 \\
\hline $\begin{array}{l}\text { Private sector } \\
\text { Government sector }\end{array}$ & $\begin{array}{l}12(13.48) \\
77(86.52)\end{array}$ & $\begin{array}{l}0(0.00) \\
14(100.00)\end{array}$ & \\
\hline working experience & & & 0.616 \\
\hline$<5$ years & $66(74.16)$ & $12(85.71)$ & \\
\hline $5-10$ year & $14(15.73)$ & $2(14.29)$ & \\
\hline$>10$ years & $9(10.11)$ & $0(0.00)$ & \\
\hline Dairy internet accessibility in hours & & & $0.017^{*}$ \\
\hline Yes & $89(100.00)$ & $12(85.71)$ & \\
\hline $\begin{array}{l}\text { No } \\
\text { Dairy internet accessibility for } \\
\text { health related purpose in hours }\end{array}$ & $0(0.00)$ & $2(14.29)$ & 0.229 \\
\hline$<2$ & $35(40.23)$ & $8(61.54)$ & \\
\hline$>=2$ & $52(59.77)$ & $5(38.46)$ & \\
\hline
\end{tabular}

Statistical test applied: Fisher's exact test.

* indicates statistically significant difference at $p \leq 0.05$.

Association between practices of dental professionals with knowledge and attitude of dental professionals.

To assess the association of teledentistry practice of dental professionals with their knowledge and attitude, the questions asked about practice were combined into summary scores. One way ANOVA has been performed and the results revealed an association of teledentistry practice with knowledge and attitude statistically $(p<0.05)$
Table 6. Correlation between teledentistry practices of dental professionals with their knowledge and attitude.

\begin{tabular}{lcccccccc}
\hline & \multicolumn{3}{c}{ Knowledge } & \multicolumn{4}{c}{ Attitude } \\
Practice & Mean & SD & Freq. & P value & Mean & SD & Freq. & P value \\
\hline Yes & 8.142 & 2.824 & 89 & \multirow{0.001*}{*}{11.966} & 2.866 & 89 & \multirow{2}{*}{.038* } \\
No & 8.662 & 1.522 & 14 & & 10.142 & 3.919 & 14 & \\
Total & 8.592 & 1.745 & 103 & & 11.718 & 3.072 & 103 & \\
\hline
\end{tabular}

Statistical test applied: One way ANOVA

* indicates statistically significant difference at $p \leq 0.05$.

\section{Discussion}

Knowledge of dental professionals about the benefits and application of teledentistry.

The present study has revealed that teledentistry benefits and applications are known by many dental professionals in Kigali-Rwanda. Most of respondents agreed or strongly agreed with the statements regarding the benefits and application of teledentistry. A large number $92(89.3 \%)$ of dental professionals in this study agreed that teledentistry can be used in every branch of dentistry like oral medicine, oral radiology,orthodontics, endodontics, prosthodontics, periodontics, maxillofacial surgery, preventive dentistry and dental education. Our results are similarly reported elsewhere (Bhambal, Saxena, \& Balsaraf, 2010a; Mihailovic et al., 2011). However, these results are a little different from those found in a study done in Udaipur in India in 2009 where only less than half $(46.7 \%)$ of dentists agreed with the statement "teledentistry can be used in every branch of dentistry" (Nagarajappa et al., 2013a). This difference might be due to the fact that in Rwanda, the information and communication technology has reached an advanced level in health sector (Ministry of Youth and ICT, 2012).

Teledentistry potentiality is also seen in dental education (Bhambal et al., 2010a; Chen, Hobdell, Dunn, Johnson, \& Zhang, 2003a). In the present study, majority 88 (85.4) of respondents agreed that teledentistry is used for education and training of primary healthcare dentists. Also majority $95(92.2 \%)$ of participants agreed that teledentistry can be used as a good tool for education to undergraduate students, post-graduate students and continuing updates of dental practitioners. The similar results have been found in India where a big proportion (58\%) of dentists agreed with the statement that teledentistry is good for dental education over internet and for training of dentists (Nagarajappa et al., 2013a).

Bhambal and colleagues also supported that undergraduate students and post graduate students can be well educated through teledentistry, they also stated that continuing professional education is easily provided to dental professionals through teledentistry and that the number of dentals specialist trainees is increased through opportunities provided by teledentistry (Bhambal et al., 2010a). Jain and colleagues also emphasized that teledentistry is a good tool to upgrade dental professionals' degree (Jain et al., 2013a). 
In Rwanda information technology have already started to be used in education even in primary schools where one laptop per child program has achieved a lot in the lives of Rwandan pupils (Ministry of Youth and ICT, 2012). Also e-learning programs are being used in some nursing school to train nurses (Frasier et al., 2008). The UR- CMHS in partnership with Telehealth center of University of Virginia (UVA) have developed a strong telehealth infrastructure which will help measure teaching hospital in country and international provision of Continuous Professional Development ( CPDs) in medical and health subjects (URCMHS, 2017). However, in Rwanda there is no evidence showing the use of teledentistry in education.

In Rwanda there is a big number of dental professionals without postgraduate study (Seymour et al., 2013a). The results of this study have also shown that only $5(4.9 \%)$ of dental professionals have done postgraduate study. The present study has also revealed a shortage of specialised dental professionals. Majority of respondents were dental professionals without post-graduation 98 (95.2\%). Based on the literatures, teledentistry may contribute a lot to upgrade the degrees of dental professionals, thereby resolving the problem of shortage of dental personnel (Nagarajappa et al., 2013a). Through teledentistry, dental professionals in remote areas are able to communicate with specialists or more skilled dental practitioners at distance (Glassman, Helgeson, \& Kattlove, 2012b).

Majority $86(83.5 \%)$ of respondents in this study, agreed with the statement "teledentistry can decrease the isolation of dental practitioners by providing peer contact specialist". Jain et al., (2013a) has supported this finding. They emphasized that having peer contact specialist is one important aspect of teledentistry as it breaks the isolation barrier among dental practitioners. Nutalapati, Boyapati, Jampani, \& Dontula (2011) also supported that through real time consultation or through store and forward teledentistry, dental professionals were able to share any information pertinent to their career thereby decreasing the isolation among those located in remote area.

In most of developing countries, most specialist dental services are found in urban area and especially most dental specialists and more skilled dental professionals are located in cities (Berndt et al., 2008). Teledentistry decreases the isolation of dental professionals in remote area as emphasized by literatures (Sanjeev, \& Shushant K, 2011a). The results of this study have also revealed that majority of respondents agreed with the statements "teledentistry can be useful in diagnosis and management of oral diseases $87(84.5 \%)$, teledentistry can be useful in early consultation of oral-facial disorders with specialists 88 (85.4\%), teledentistry can enable dental practitioners to make sound decisions regarding management of oral diseases and referral to appropriate dental specialists $90(87.4 \%)$, and that teledentistry prevents doctors' mistakes and improves costeffectiveness of dental treatment $77(75 \%)$ ". Those results are supported by different published literatures and researches. They support that speed, cost-effectiveness, simplicity and maximum therapeutic gain are some of teledentistry benefits for consultation, diagnosis and management of oral diseases and oral-facial disorders (Nagarajappa et al., 2013b); (Jain et al., 2013b);(Rao, Singh, Chandra, \& Gupta, 2012);(Bhambal, Saxena, \& Balsaraf, 2010b);(Sanjeev, \& Shushant K, 2011b);(Chhabra, Chhabra, Jain, Kaur, \& Bansal, 2011).

In addition, teledentistry prevents dental practitioners' mistakes and enable them to make sound decisions (Panat, Chakarvarty, \& Aggarwal Ashish, 2012). In Rwanda there is shortage of dental practitioners especially dental specialists of all kinds (Seymour et al., 2013b). Oral diseases and oral -facial disorders are among dental problems that may impinge the lives of people (Jain et al., 2013b).

Due to shortage of qualified dental specialists to diagnose and treat these disorders in early stage, Rwandan population with oral diseases may lose their lives due to lack or delay of diagnosis and management of those oral problems (Seymour et al., 2013b). The results of this study have shown that dental professional in Kigali-Rwanda are aware that teledentistry can help to overcome this problem by providing early and easy consultation of oralfacial disorders with specialists. If teledentistry could be adopted and used for consultation of oral- facial disorders with available specialists, the oral - facial disorders which impinge the lives of people in Rwanda could be reduced.

\section{Attitude of dental professional towards the benefits and application of teledentistry.}

In developing countries the number of general dentists and dental specialists per person is still low (Ozkan Ata, \& Ozkan, 2009b). Through applications provided by teledentistry, the existing dental professionals in those countries should be used effectively to be able to serve their population. In the present study the respondents agreed with the statements "teledentistry can save them time $80(79.2 \%)$ and money $71(69 \%)$.

The results of the current study are similar to the results of a study than in Turkey in 2009 where 181 (83\%) anticipated that teledentistry would save time and $157(72 \%)$ anticipated that teledentistry would save money (Ozkan Ata, \& Ozkan, 2009). Other different literatures have supported teledentistry as a mean to save money and to save time (Jain et al., 2013b).

However the currents results are different from the results of a research done by Nagarajappa and colleagues in Udaipur, India where only 35 (33.3\%) agree with the statement "teledentistry can save me time" and only $22(21 \%)$ agree with the statement "teledentistry can reduce costs for the dental practices" (Nagarajappa et al., 2013b).

Teledentistry is essential for monitoring of patients (telemonitoring; Ozkan Ata, \& Ozkan, 2009). Majority of 
respondents $80(77.7 \%)$ perceived teledentistry to be useful for monitoring of patients' conditions. A study done in Turkey in 2009 has shown similar results where majority 178 (81\%) of respondents were all positive that using teledentistry, they would be able to monitor patients' conditions (Ozkan Ata, \& Ozkan, 2009b). However different results have been found by (Nagarajappa et al., 2013) in a research done in India in 2013 where only $47(44.8)$ respondents were positive with the statement "using teledentistry, I will be able to monitor my patient's condition well".

Different applications of teledentistry for dental education have been emphasized by different literatures (Chhabra et al., 2011; Bhambal et al., 2010b; Jain et al., 2013b; Chen et al., 2003). In the present study, majority 94 (91.2\%) of dental professionals thought that through videoconference (a medium of teledentistry) students can be educated and staff be trained effectively. A study done in Turkey has also revealed that majority 206 (94\%) of respondents agreed that teledentistry is good for dental education (Ozkan Ata, \& Ozkan, 2009b). Teledentistry has been shown to be efficient to provide low cost, real-time interaction between students and instructors. Different literatures emphasize the effectiveness videoconference for training dental practitioners, dental students, dental assistants and dental office staff (Eaton, Francis, Odell, Reynolds, \& Mason, 2001); (Chhabra et al., 2011). Through videoconference, universities can also collaborate to exchange and share experience among their staff which contribute to quality dental care services (Reynolds, Eaton, \& Mason, 2008) videoconferencing has been further extended to support distance learning and has been evaluated through a number of educational projects. The use of the technology has been integrated as a core method of delivering training and education at all levels in dentistry. Advances in systems, services and standards have produced equipment and telecommunications links that are userfriendly, reliable, affordable and offer better quality image resolution. In addition, a wide range of peripheral devices can be connected to basic videoconferencing equipment to introduce a variety of teaching aids and material into a session. Projects such as PROVIDENT (Postgraduate Regional Online Videoconferencing in Dentistry.

Majority of dental professionals practicing dentistry outside the city hold Bachelors of dental therapy or advanced diploma in dentistry (Seymour et al., 2013). The use of videoconference, a medium of teledentistry would be very beneficial to allow the population outside the city to access specialty dental services. Again dental professionals working in those areas would gain more skills and knowledge through Continuing Professional Development provided by teledentistry applications.

Teledentistry has been shown to have endless potential (Bhambal et al., 2010b). Different literatures have emphasized the potentiality of teledentistry in different areas of dentistry (Bhambal et al., 2010; Nagarajappa et al., 2013b). Majority 97 (94.2\%) of respondents overall believe that teledentistry is potential and has to be integrated into their current practices. The same results have been revealed by a study done in Turkey in 2009 where 212 (97\%) agreed or strongly agreed that teledentistry is potential and has to be integrated into their current practices (Ozkan Ata, \& Ozkan, 2009b). This is an indicator that dental professionals generally understand and would like to use teledentistry in their daily activities.

\section{Practices of dental professionals towards teledentistry.} Teledentistry has been defined as the use of telecommunication technology and internet in dental field (Sanjeev, \& Shushant K, 2011b). At its low level, practicing teledentistry has been said to be as simple as turning on the television or making a phone call (Sanjeev, \& Shushant K, 2011b).

In the present study, majority $87(84.5 \%)$ of respondents consult or communicate with other dental professionals through internet or mobile phone. Regarding category of people with whom they interact, 30 (29.1\%) intercommunicate with patients; $52(50.5 \%)$ with students; 29 (28\%) with educators; $32(31 \%)$ with specialists; 26 with own peer and only 4 (4\%) communicate with insurance companies. The results revealed that the means of intercommunication were email $69(67 \%)$; phone call and sms 21 (20.4\%); Facebook, linkedin and whatsapp $13(12.6 \%)$ while videoconference showed only $7(6 \%)$. Regarding reasons for intercommunication 43 (42\%) intercommunicate to share patient information, $40(39 \%)$ intercommunicate to discuss a patient case, $37(36 \%)$ intercommunicate for course materials clarification; 26 (25\%) intercommunicate to get opinion from an expert, $23(22.3 \%)$ intercommunicate for patient follow-up. Only a small number of dental professionals are sharing digital $\mathrm{x}$-ray image $13(12.6 \%)$ or digital photographs $8(7.8 \%)$ as a reason of intercommunication.

Based on published literatures (Sanjeev, \& Shushant K, 2011b), dental professionals in Kigali- Rwanda are practicing teledentistry at low level. At its highest level teledentistry involved more sophisticated applications like real time interaction, e.g. in projects like PROVIDENT (Postgraduate Regional Online Videoconferencing in Dentistry) and DUET (Dental Undergraduate Education by Teleconferencing) teledentistry, all of which have shown positive impact on education and continuing development of dental professionals (Reynolds et al., 2008)videoconferencing has been further extended to support distance learning and has been evaluated through a number of educational projects. The use of the technology has been integrated as a core method of delivering training and education at all levels in dentistry. Advances in systems, services and standards have produced equipment and telecommunications links that are user-friendly, reliable, affordable and offer better quality image resolution. In addition, a wide range of peripheral devices can be connected to basic videoconferencing equipment to introduce a variety of teaching aids and 
material into a session. Projects such as PROVIDENT (Postgraduate Regional Online Videoconferencing in Dentistry.

These results are similar to the results of a study done by Ignatus (2013). The objective was to determine what type of electronic consultation tool and methods used for dental consultation and patient-provider communication. The questionnaire has been sent to 120 dental practitioners in public and private sector. The results showed that majority $(53 \%)$ used email for consultation and only $10 \%$ used videoconference for consultation (Ignatius, 2013).

We found that there is a significant association $(P<0.05)$ between practice with knowledge, attitude and internet access. William and colleague found similar association.

They have emphasized that knowledge and attitude can influence the adoption of technology in healthcare (William \& Dickinson, 2010). Peter has also supported that the practice of teledentistry goes together with the use of telecommunication and internet accessibility and that internet accessibility and usage is the source of different opportunities to human being when used in meaningful and positive way. He also said that, learning, gaining new experience and skills, acquiring new developing knowledge, are all benefits from internet access and usage (Peter, 2010). Teledentistry practice depends also on the availability of internet as stated in different literatures (Sanjeev, \& Shushant K, 2011a). In Rwanda the internet availability has reached a high level compared to other low income countries in Africa continent (Ministry of Youth and ICT, 2012). The findings of the current study are indicator that, if adopted, teledentistry can bring dental services provided in Rwanda to another step.

\section{Conclusion}

The results of this study revealed that dental professionals are knowledgeable and have positive attitude towards teledentistry. Again, dental professionals overall believe that teledentistry is good and it has to be integrated into the current dental practice. However, the practice of teledentistry of dental professionals is still quite at low level compared to other advanced applications of teledentistry done in other areas as shown in different literatures and researches. Therefore new policies and programs should be designed to ensure the implementation of teledentistry and to allow its use at advanced level as shown in the literature. To bring dental practice to another level in areas like dental education, dental research, community outreaches, and dental health care delivery, there is a need to use the opportunities provided by teledentistry technology. Further similar research is important which may include the rural area and the bigger sample size.

\section{Conflicts of interest}

The author declares that there is no conflict of interest in that paper

\section{Author's contribution}

JM conceived the present study, implemented it and wrote the manuscript, KN, IM, UP, NT, contributed for manuscripts drafting and finalization.

\section{Acknowledgement}

Thanks to all dental professionals who participated in this study.

\section{References}

Berndt, J., Leone, P., \& King, G. (2008). Using teledentistry to provide interceptive orthodontic services to disadvantaged children. American Journal of Orthodontics and Dentofacial Orthopedics, 134(5), 700-706. https://doi.org/10.1016/j.ajodo.2007.12.023

Bhambal, A., Saxena, S., \& Balsaraf, S. V. (2010a). Teledentistry: potentials unexplored! J Int Oral Health, 2, 1-6.

Bhambal, A., Saxena, S., \& Balsaraf, S. V. (2010b). Teledentistry: potentials unexplored! J Int Oral Health, 2, 1-6.

Chen, J.-W., Hobdell, M. H., Dunn, K., Johnson, K. A., \& Zhang, J. (2003a). Teledentistry and its use in dental education. Journal of the American Dental Association (1939), 134(3), 342-346.

Chen, J.-W., Hobdell, M. H., Dunn, K., Johnson, K. A., \& Zhang, J. (2003b). Teledentistry and its use in dental education. Journal of the American Dental Association (1939), 134(3), 342-346.

Chhabra, N., Chhabra, A., Jain, R. L., Kaur, H., \& Bansal, S. (2011). Role Of Teledentistry In Dental Education: Need Of The Era.

Cooper, B., \& Engeswick, L. (2007). Knowledge, attitudes, and confidence levesl of dental hygiene students regarding teledentistry: A pilot study. Internet $J$ Allied Health Sci Practice, 5(4). Retrieved from http://ijahsp.nova.edu/articles/vol5num4/pdf/cooper.pdf

Costa, A. L. P. da, Silva, A. A., \& Pereira, C. B. (2011). Teleortodontia: ferramenta de auxílio à prática clínica e à educação continuada. Dental Press Journal of Orthodontics, 16(6), 15-21. https://doi. org/10.1590/S2176-94512011000600003

Eaton, K. A., Francis, C. A., Odell, E. W., Reynolds, P. A., \& Mason, R. D. (2001). Participating dentists' assessment of the pilot regional online videoconferencing in dentistry (PROVIDENT) project. British Dental Journal, 191(6), 330-335.

Frasier, H., May, M. A., \& Wanchoo, R. (2008). e-Health Rwanda Case Study.

Glassman, P., Helgeson, M., \& Kattlove, J. (2012a). Using telehealth technologies to improve oral health for vulnerable and underserved populations. Journal of the California Dental Association, 40(7), 579-585.

Glassman, P., Helgeson, M., \& Kattlove, J. (2012b). Using telehealth technologies to improve oral health for 
vulnerable and underserved populations. CA Dent Assoc J, 40(7), 579-85.

Ignace Gatare. (2011). National ICT Strategy and plan $\mathrm{NICl}$ III. Ministry of ICT iN THE office of the presedent.

Ignatius, E. (2013). Use and Attitudes towards Teleconsultation in Dentistry. E-Health Telecommunication Systems and Networks, 02(01), 9-12. https://doi. org/10.4236/etsn.2013.21002

Jain, A., Bhaskar, D. J., Gupta, D., Agali, C., Gupta, V., \& Karim, B. (2013a). Teledentistry: Upcoming Trend in Dentistry. Med Dent Scie, 1(2), 112-115.

Jain, A., Bhaskar, D. J., Gupta, D., Agali, C., Gupta, V., \& Karim, B. (2013b). Teledentistry: Upcoming Trend in Dentistry. Med Dent Scie, 1(2), 112-115.

Mariño, R., \& Ghanim, A. (2013). Teledentistry: a systematic review of the literature. Journal of Telemedicine and Telecare, 19(4), 179-183. https://doi. org/10.1177/1357633X13479704

Mihailovic, B., Miladinovic, M., \& Vujicic, B. (2011). Telemedicine in Dentistry (Teledentistry). Advances in Telemedicine: Applications in Various Medical Disciplines and Geographical Areas, 25-30.

Ministry of Youth and ICT. (2012). Measuring ICT sector performance and Tracking ICT for Development (ICT4D) towards Rwanda Socio-Economic Transformation.

Nagarajappa, R., Aapaliya, P., Sharda, A. J., Asawa, K., Tak, M., Pujara, P., \& Bhanushali, N. (2013a). Teledentistry: Knowledge and Attitudes among Dentists in Udaipur, India. Oral Health Dent Manag, 12(502), 2.

Nagarajappa, R., Aapaliya, P., Sharda, A. J., Asawa, K., Tak, M., Pujara, P., \& Bhanushali, N. (2013b). Teledentistry: Knowledge and Attitudes among Dentists in Udaipur, India. Oral Health Dent Manag, 12(502), 2.

Nutalapati, R., Boyapati, R., Jampani, N., \& Dontula, B. S. K. (2011). Applications of teledentistry: A literature review and update. Journal of International Society of Preventive and Community Dentistry, 1(2), 37. https://doi.org/10.4103/2231-0762.97695

Ozkan Ata, S., \& Ozkan, S. (2009a). Information Technology in Oral Health Care: Attitudes of Dental Professionals on the Use of Teledentistry in Turkey. Presented at the European and Mediterranean Conference on Information Systems, Crowne Plaza Hotel, Izmir: EMCIS2009.

Ozkan Ata, S., \& Ozkan, S. (2009b). Information Technology in Oral Health Care: Attitudes of Dental Professionals on the Use of Teledentistry in Turkey. Crowne Plaza Hotel, Izmir: EMCIS2009.

Panat, S. ., Chakarvarty, A., \& Aggarwal Ashish. (2012). TELEDENTISTRY: A NEW REVOLUTION. Journal Od Dental Science and Oral Reabilitation.

Peter, J. (2010). Equality and Human Rights Commission Triennial Review: Education (Lifelong Learning). School of Education University of Southampton.
Rao, J., Singh, K., Chandra, G., \& Gupta, K. (2012). Teledentistry in India: Time to deliver. Journal of Education and Ethics in Dentistry, 2(2), 61. https://doi.org/10.4103/0974-7761.121258

Reynolds, P. A., Eaton, K. A., \& Mason, R. (2008). Seeing is believing: dental education benefits from developments in videoconferencing. British Dental Journal, 204(2), 87-92. https://doi.org/10.1038/ bdj.2008.9

Sanjeev, M., \& Shushant K, G. (2011a). Teledentistry a new trend in oral health. International Journal of Clinical Cases and Investigations, 2(6), 49-53.

Sanjeev, M., \& Shushant K, G. (2011b). Teledentistry a new trend in oral health. International Journal of Clinical Cases and Investigations, 2(6), 49-53.

Seymour, B., Muhumuza, I., Mumena, C., Isyagi, M., Barrow, J., \& Meeks, V. (2013a). Including oral health training in a health system strengthening program in Rwanda. Global Health Action, 6(201), 9. https://doi.org/http://dx.doi.org/10.3402/gha. v6i0.20109

Seymour, B., Muhumuza, I., Mumena, C., Isyagi, M., Barrow, J., \& Meeks, V. (2013b). Including oral health training in a health system strengthening program in Rwanda. Global Health Action, 6(201), 9. https://doi.org/http://dx.doi.org/10.3402/gha. v6i0.20109

UR-CMHS. (2017). Towards the strengthened e-health and telemedicine delivery in the College of Medicine and Health Sciences. Retrieved from http:// www.cmhs.ur.ac.rw/news-details/article/284/

William, L., \& Dickinson, H. (2010). Can knowledge management enhance technology adoption in health care?, 6(3), 309-331. 